\title{
Physicochemical Characterization of Finasteride Nanosystem for Enhanced Topical Delivery
}

This article was published in the following Dove Press journal:

International Journal of Nanomedicine

\author{
Malik Muhammad Irfan (iD) ${ }^{1,2}$ \\ Shefaat Ullah Shah $\mathbb{D}^{1,2}$ \\ Ikram Ullah Khan $\mathbb{D}^{3}$ \\ Muhammad Usman Munir ${ }^{4}{ }^{4}$ \\ Nauman Rahim Khan (D) ${ }^{1,2}$ \\ Kifayat Ullah Shah' \\ Saif Ur Rehman ${ }^{5}$ \\ Muhammad Sohaib ${ }^{1,2}$ \\ Hafiz Muhammad Basit ${ }^{1,2}$ \\ Saima Mahmood ${ }^{1,2}$
}

'Department of Pharmaceutics, Faculty of Pharmacy, Gomal University, D.I. Khan,

29050, KPK, Pakistan; ${ }^{2}$ Gomal Centre for

Skin/Regenerative Medicine and Drug

Delivery Research (GCSRDDR), Faculty

of Pharmacy, Gomal University, D.I. Khan,

29050, KPK, Pakistan; ${ }^{3}$ Department of

Pharmaceutics, Faculty of Pharmaceutical

Sciences, Government College

University, Faisalabad, Pakistan;

${ }^{4}$ Department of Pharmaceutical

Chemistry, College of Pharmacy, Jouf

University Sakaka, Aljouf, 72388, Saudi

Arabia; ${ }^{5}$ Department of Pharmacy,

Faculty of Medical and Health Sciences, University of Poonch, Rawlakot, AJK, Pakistan
Correspondence: Shefaat Ullah Shah Department of Pharmaceutics, Faculty of Pharmacy, Gomal University, D.I. Khan, Pakistan

Tel +92-336-5I40682

Email shefaatbu@gmail.com
Introduction: The current work aimed to formulate a novel chitosan-based finasteride nanosystem (FNS-NS) for skin delivery to optimize the drug availability in skin for a longer time and enhance ex vivo performance of finasteride against androgenic alopecia.

Methods: Both undecorated and chitosan decorated FNS-NSs were synthesized by a high energy emulsification technique. All the prepared nanosystems were further subjected to physicochemical characterizations like $\mathrm{pH}$, viscosity, encapsulation efficiency, surface morphology and in vitro drug release behavior. The influence of the nanosystem on the drug permeation and retention in rat skin was examined using Franz diffusion cell apparatus.

Results: The droplet size of developed nanosystems ranged from 41 to $864 \mathrm{~nm}$ with a low polydispersity index. The zeta potential of the nanosystems was between $-10 \mathrm{mV}$ and +56 $\mathrm{mV}$. This chitosan decorated nanosystem exhibited controlled drug release, ie about $78-97 \%$ in $24 \mathrm{~h}$. Among all the nanosystems, our chitosan decorated formulation (F5) had low drug permeation $\left(16.35 \mu \mathrm{g} / \mathrm{cm}^{2}\right)$ and higher drug retention $\left(10.81 \mu \mathrm{g} / \mathrm{cm}^{2}\right)$.

Conclusion: The abovementioned results demonstrate satisfactory in vitro drug release, skin retention profiles and ex vivo performance with chitosan decorated FNS-NS (F5). This optimized formulation could increase drug availability in skin and could become a promising carrier for topical delivery to treat androgenic alopecia.

Keywords: finasteride, nanosystem, solubility, chitosan, permeation, retention, androgenic alopecia

\section{Introduction}

Androgenic alopecia is a very common skin disorder affecting generally the male population. This dermatological disease is characterized by a continuous reduction of hairs from the scalp region. ${ }^{1}$ An endogenous hormone called testosterone produced by the adrenal gland seems to play a significant role in androgenic alopecia. In human beings, an enzyme $5 \alpha$ reductase enzyme is mainly responsible for the conversion of testosterone into dihydrotestosterone. Generally, two types of $5 \alpha$ reductase enzymes are found in the human tissues, ie type I and II. ${ }^{2}$ Type I is present in the skin and outer sheath of the hair follicles, whereas the type II enzyme was mostly evident in the prostate and seminal vesicle. The type II enzyme has been described as the major cause of androgenic alopecia and was apparent in dermal papillae and the outer sheath of the hair follicle root. ${ }^{3}$

Finasteride (FNS) is a $5 \alpha$ reductase inhibitor and belongs to a 4-aza 3-oxosteroid compound. It was initially introduced for the management of benign prostatic hyperplasia ( $5 \mathrm{mg} /$ day) and later approved and marketed for androgenic alopecia with a daily dose of $1 \mathrm{mg}{ }^{4,5}$ Recently, researchers have established the role of 
finasteride in melanogenesis by inhibiting melanocortin-1 receptor $(\mathrm{MC} 1 \mathrm{R})$ in melanoma cells and melanocytes. ${ }^{6}$ FNS is a highly lipophilic compound ${ }^{7}$ and an analog of androgen steroidal hormones like testosterone and dihydrotestosterone. ${ }^{8,9}$ The FNS exerts its action by inhibiting type II $5 \alpha$ reductase enzyme. ${ }^{10}$ The adverse events reported with oral intake of FNS are sexual disorders such as impotency, ejaculation disorder, erectile dysfunction and mental impairment. ${ }^{11}$ Alongside, some minor side effects reported are gynecomastia, lowering of temperature, fatigue and weight gain. ${ }^{12,13}$ FNS belongs to the Biopharmaceutical Classification System (BCS) class II drugs and has high permeability and poor aqueous solubility. ${ }^{14}$ Because of the high molecular weight of FNS, ${ }^{15}$ it is poorly penetrated through the stratum corneum (SC) of the skin, which constitutes a lipid barrier, and thus these inherent aspects do not allow permeation of FNS. The skin, serving as the largest organ, protects the body from the entry of various hazardous materials ${ }^{16}$ and has three distinctive layers; the epidermis, dermis and hypodermis. The top layer of the skin is comprised of the stratum corneum which acts as a main physical barrier for drug penetration and permeation through the skin. ${ }^{17}$ In the past, several FNS topical delivery systems were reported including polymersome, vesicular nanocarriers (ethosome and liposome), liquid crystalline nanoparticles, $0.25 \%$ solution, polymeric nanoparticles, matrix system and liposomal gel, etc. ${ }^{18-24}$

There is a growing interest in nanoemulsion based drug delivery systems for lipophilic bioactive components such as antimicrobial agents, anti-inflammatory agents, vitamins and anti-cancerous agents. The introduction of bioactive components into the emulsion enhances their physical and chemical durability, provides functional properties and increases systemic bioavailability and transdermal permeability which influence the characteristics of their end-use in medicinal products or cosmetics. ${ }^{25}$ However, to the best of our knowledge, FNS chitosan decorated nanoemulsions also known as nanosystems (NSs) for improved performance through skin delivery have not been yet investigated. The NS was chosen as a drug carrier since this system has been commonly used to enhance the delivery of hydrophobic drugs to the skin. ${ }^{26}$

NSs are biphasic systems having submicron droplets (1-1000 nm) stabilized by emulgents forming a single phase. ${ }^{27}$ Several research groups and pharmaceutical companies have become progressively interested in the design and development of novel nanosystem formulations with unique features, ie high colloidal stability, delayed release pattern of the molecule with appropriate droplet size and structure, improved permeation, better biocompatibility, efficient entrapment efficiency along with cost effectiveness and scalability features. ${ }^{28,29}$ Almost $40 \%$ of the drugs have lower aqueous solubility and their solubility can be enhanced with the development of NSs. ${ }^{30}$

These unique characteristics make NSs an attractive carrier for applications in various fields such as pharmaceuticals, cosmetics and food as well as in drug delivery applications. ${ }^{31}$ Moreover, chitosan based drug nanocarriers have been extensively studied for their biomedical use in many targeted sites like skin, brain, liver and tumor cells. ${ }^{32-36}$ These findings will open the platform to formulate novel chitosan based NSs for enhanced skin delivery of FNS. In the current study, we hypothesized to establish an NS for effective delivery of FNS across the skin.

However, some stability issues like creaming, flocculation, sedimentation and Ostwald ripening occur due to the presence of inappropriate amounts of surfactants in the nanosystems. ${ }^{37}$ Biopolymers like proteins and polysaccharides (chitosan and alginate, etc.) are used for coating of nanosystems. Chitosan has previously shown tremendous potential to improve stability and surface characteristics and prevent aggregation of droplets by maintaining both steric repulsion and electrostatic interactions. ${ }^{38}$ Chitosan is a non-toxic, biodegradable and biocompatible polymer that has mucoadhesive properties and thus could be used in many formulations for surface decoration of nanoemulsions. $^{25,39,40}$ The chitosan based drug carrier increases the propensity of drug diffusion through the layers of the peripheral skin. The effectiveness of the penetration and retention of skin drugs has been reported to be assisted by the chitosan based particulate material. $^{29,41}$

The aim of the current study was to investigate the local retention of the drug at the targeted site of the skin and its release profiles from different formulations. Moreover, the influence of chitosan on the drug retention in the skin, in vitro and ex vivo drug release behavior was also investigated.

\section{Materials and Methods}

\section{Materials}

Finasteride was gifted by Ferozsons Laboratories, Nowshera, Pakistan. Chitosan (low molecular weight), 
oleic acid, Tween 80 and Span 80 were purchased from Sigma-Aldrich, USA. Male Sprague Dawley rats were purchased from Peshawar University, Pakistan. All other chemicals used were of analytical grade.

\section{Solubility of Finasteride}

To determine the FNS solubility in different oils, an excess amount of the drug was added into a separate capped glass vial containing $2 \mathrm{~mL}$ of oil (ie oleic acid, olive oil and eucalyptus oil). The mixtures were vortex for $5 \mathrm{~min}$ and placed in an isothermal shaking water incubator (WNB14; Memmert $\mathrm{GmbH}+\mathrm{Co}$. KG, Germany) at $37{ }^{\circ} \mathrm{C}$ for $72 \mathrm{~h}$ to maintain equilibrium. All the samples were centrifuged (SCILOGEX, D3024) at 10,000 rpm for $10 \mathrm{~min}$. Finally, the amount of FNS was determined by UV/visible spectrophotometer (UV-1800 240 V; Shimadzu Corporation, Japan) at a wavelength $\left(\lambda_{\max }\right)$ of $210 \mathrm{~nm}$ using a previously constructed calibration curve in acetate buffer solution (pH 5.5).

\section{Preparation of Finasteride NSs}

FNS-NSs were prepared by a high energy emulsification technique as described by Bakshi et al, with minor modifications. ${ }^{42}$ The NSs formulations are shown in Table 1 . Briefly, blank NS was prepared by gently mixing oleic acid and Span 80 for 10 min with continuous stirring at $30{ }^{\circ} \mathrm{C}$. Acetone was added to the above mixture and was stirred for an additional $30 \mathrm{~min}$. In the second step, an aqueous phase was prepared by mixing Tween 80 with distilled water and the mixture was left stirring at $30{ }^{\circ} \mathrm{C}$ for 30 $\mathrm{min}$. In the final step, the oily phase was incorporated into the aqueous phase dropwise with continuous stirring for $45 \mathrm{~min}$. The resultant mixture was subjected to a high pressure homogenizer at $10,000 \mathrm{psi}$ for $2 \mathrm{~min}$. Drug-loaded NSs were prepared by employing a similar procedure except the drug was first dissolved in oleic acid with continuous stirring for $10 \mathrm{~min}$.

\section{Chitosan Decorated NSs}

FNS-NSs (F2 to F5) were decorated with different concentrations of chitosan $(0.125$ to $1 \% \mathrm{w} / \mathrm{w})$ to enhance skin retention of the drug with reduced permeation. Briefly, $1 \%$ acetic acid was used to dissolve the polymer. The resulting $\mathrm{pH}$ of the formulation dropped from 5.1 to 3.42 (acidic) and later was adjusted with $0.2 \mathrm{M}$ sodium hydroxide solution to meet the skin physiological $\mathrm{pH}$. This polymeric solution was mixed with Tween 80 in water by stirring at $30{ }^{\circ} \mathrm{C}$ for $30 \mathrm{~min}$. The oil phase was prepared using the same method as described for drug-loaded NSs. The rest of the process was the same as used for undecorated NSs.

\section{Entrapment Efficiency}

A method reported by Loureiro Contente et al ${ }^{43}$ was adopted with few modifications to determine the entrapment efficiency (E.E). Briefly, $1 \mathrm{~mL}$ of each formulation was taken in Eppendorf tubes and centrifuged for $30 \mathrm{~min}$ at $10,000 \mathrm{rpm}$. The supernatant layer of the separated formulation was diluted with $3 \mathrm{~mL}$ of ethanol. The sediment portion was subsequently dissolved into $3 \mathrm{~mL}$ of ethanol. The drug was quantified by measuring absorbance at $210 \mathrm{~nm}\left(\lambda_{\max }\right)$ using the UV/visible spectrophotometer and the results were calculated from a previously constructed FNS calibration curve in ethanol. All the experiments were carried out in triplicate. The \% E.E was calculated by the following equation:

$$
\% E . E=\frac{\text { Total drug }- \text { free drug }}{\text { Total drug }} * 100
$$

\section{Thermodynamic Stability}

Although nanoemulsions are reported to be thermodynamically/thermokinetically stable, dispersion stability studies are required under various circumstances. Thermodynamic stability studies were done using three different methods as given below. ${ }^{44,45}$

Table I Microstructure and Composition of Various Developed Formulations of Finasteride Nanosystems

\begin{tabular}{|l|l|l|l|l|l|l|l|l|}
\hline Formulation & Microstructure & \multicolumn{6}{l|}{ Composition (\% w/w) } \\
\hline & & Drug & Oleic Acid & Span 80 & Acetone & Tween 80 & Chitosan & Water \\
\hline F0 (blank) & Oil in water & - & 0.77 & 0.154 & 0.05 & 0.154 & - & 98.872 \\
FI & Oil in water & 0.05 & 0.77 & 0.154 & 0.05 & 0.154 & - & 98.822 \\
F2 & Oil in water & 0.05 & 0.77 & 0.154 & 0.05 & 0.154 & 0.125 & 98.697 \\
F3 & Oil in water & 0.05 & 0.77 & 0.154 & 0.05 & 0.154 & 0.25 & 98.572 \\
F4 & Oil in water & 0.05 & 0.77 & 0.154 & 0.05 & 0.154 & 0.5 & 98.322 \\
F5 & Oil in water & 0.05 & 0.77 & 0.154 & 0.05 & 0.154 & 1 & 97.822 \\
\hline
\end{tabular}




\section{Cooling Thaw Cycle}

In this method, a specific quantity of each formulation was kept in a capped vial which was then placed in a refrigerator at $-21^{\circ} \mathrm{C}$ for $24 \mathrm{~h}$. The visual characteristics of each formulation were checked in triplicate after returning to their original liquid state at room temperature. ${ }^{44,45}$

\section{Centrifugation Test}

NSs after passing through cool and thaw steps were subjected to centrifugation. All the samples were centrifuged at $10,000 \mathrm{rpm}$ for $10 \mathrm{~min}$ to check any turbidity or phase change. ${ }^{44,45}$

\section{Heating and Cooling Thaw Cycle}

Furthermore, upon completion of the centrifugation test, all centrifuged samples were exposed to the heating and cooling thaw cycle. For the cooling cycle, all samples were placed at $4{ }^{\circ} \mathrm{C}$ in the refrigerator and for the heating thaw cycle (at $45^{\circ} \mathrm{C}$ ) in an incubator for a period of $48 \mathrm{~h}$. Lastly, the physical stability of the FNS-NSs was examined. ${ }^{44,45}$

We have only introduced thermodynamically stable preparations for further characterization and evaluation.

\section{Characterization of NSs}

All NSs were characterized in terms of their particle size, polydispersity index (PDI), zeta potential, $\mathrm{pH}$, specific viscosity and morphology.

\section{Particle Size, PDI and Zeta Potential}

The droplet size and PDI of FNS-NSs were measured using the photon correlation spectroscopy technique (Zetasizer Nano ZS 90; Malvern instrument, UK) at 25 ${ }^{\circ} \mathrm{C}$ in a quartz cell. ${ }^{46}$ The samples were suitably diluted (1:10) to avoid the multi-scattering phenomenon. The zeta potentials of prepared NSs were also determined using Zetasizer with a detection angle of $90^{\circ}$. The experiment was conducted in triplicate and results were averaged.

\section{$\mathrm{pH}$ and Specific Viscosity Measurement}

The $\mathrm{pH}$ and specific viscosity of all formulations were measured with a digital $\mathrm{pH}$ meter and U-tube viscometer as mentioned earlier. ${ }^{47,48}$

\section{Morphology}

The morphological features of prepared NSs were evaluated using a transmission electron microscopy (TEM; Hitachi H-6000). Briefly, $3 \mu \mathrm{L}$ of each formulation was placed on the carbon-coated grid and left for $5 \mathrm{~min}$. The grid was then blotted with filter paper and stained with $2 \%$ phosphotungstic acid. Afterward, the sample was observed at $100 \mathrm{kV}$ voltage and photographs were taken at different resolutions. $^{49}$

\section{In vitro Drug Release Studies}

Drug release from FNS-NSs was assessed through Franz Diffusion Cell apparatus (PermeGear, USA) using an artificial membrane, ie polytetrafluoroethylene (PTFE) (0.45 $\mu \mathrm{m}$ pore size; Sartorius AG, Goettingen, Germany) at $32{ }^{\circ} \mathrm{C}$. A specific quantity of each formulation was introduced in the donor compartment. Similarly, the receptor chamber was filled with a specific amount of acetate buffer as a drug release medium. Additionally, throughout the test, the sink condition was maintained along with magnetic stirring at $150 \mathrm{rpm}$. To evaluate the drug release behavior, aliquots $(500 \mu \mathrm{L})$ were withdrawn at specific time intervals $(0,0.5,1,2$, $4,8,12,16$ and $24 \mathrm{~h}$ ) and then compensated with 0.5 $\mathrm{mL}$ of fresh buffer medium kept at $32{ }^{\circ} \mathrm{C}$. The samples were analyzed to check the FNS released pattern using the UV/visible spectrophotometer at $210 \mathrm{~nm}$. The analysis was performed in triplicate to minimize any error and the results were averaged.

\section{Kinetic Models for Drug Release}

To evaluate the kinetics of drug release from the FNS-NSs, mathematical models such as zero order, first order, Higuchi and Korsmeyer--Peppas were employed to in vitro drug release data.

\section{Ex vivo Drug Permeation and Drug Retention Studies}

\section{Animal Ethics Approval and Consent to Practice}

The guidelines of the Food and Drug Administration Authority (FDA, 2005) and the Science Association of Laboratory Animals have been adopted under the permission notification number $823 / \mathrm{QEC} / \mathrm{GU}$ in compliance with the Ethical Review Committee, Gomal University, D. I. Khan (Pakistan).

\section{Skin Preparation}

Sprague Dawley rats were kept in separate cages at room temperature in the built-in animal house facility until acclimatization. Standard pellet food and free access to water were provided to rats and bedding was changed regularly. For the ex vivo studies, rats were sacrificed by the cervical dislocation method. The abdominal region of 
each rat was shaved with a sharp razor and washed with normal saline $(0.9 \% \mathrm{w} / \mathrm{v} \mathrm{NaCl})$ solution. Skin samples were surgically removed with scissors and again washed with normal saline and were wrapped in aluminum foil. The samples were stored at $-40{ }^{\circ} \mathrm{C}$ till further use. ${ }^{50}$

\section{Skin Permeation Studies}

The Franz diffusion cell (FDC) was used to analyze the permeation profile of FNS-NSs through rat skin. The receptor compartment of the FDC was filled with $6.4 \mathrm{~mL}$ of 7.4 $\mathrm{pH}$ phosphate buffer solution. The skin sample $(0.88 \mathrm{~mm}$ thickness) was thawed for $1 \mathrm{~h}$ in the receptor medium for pre-equilibration purposes at room temperature and then mounted between the donor and acceptor chambers of the FDC. After mounting, the skin was again left to equilibrate for an additional $45 \mathrm{~min}$. The skin was mounted between donor and receptor chambers in such a way that the epidermis has direct contact with the drug (facing donor chamber) and the dermis side was sunk in the buffer medium of the receptor chamber. The temperature was kept constant at $37^{\circ}$ $\mathrm{C} \pm 0.5$ to mimic the in vivo condition by a circulating interlinked water jacket and allow stirring at the speed of $250 \mathrm{rpm}$. FNS-NSs (equivalent to $1 \mathrm{mg}$ of FNS) were loaded into the donor cell covered with aluminum foil. Aliquots of $500 \mu \mathrm{L}$ were withdrawn from the acceptor chamber and replaced with the same quantity of fresh buffer solution into the sampling port of the receptor chamber sequentially at predestined time intervals (up to $24 \mathrm{~h}$ ). All samples were analyzed in triplicate by high-pressure liquid chromatography (HPLC). ${ }^{51}$

The cumulative drug permeation through excised rat membrane was calculated from the following equation:

$$
Q n=\frac{C n * V r+\sum_{i=1}^{n-1} C i * V s}{A}
$$

where Qn is the cumulative amount of FNS permeated via a unit area of the membrane $\left(\mu \mathrm{g} / \mathrm{cm}^{2}\right), \mathrm{Cn}$ is the drug concentration present in the acceptor compartment at a different time of the nth sample, $\mathrm{Ci}$ is the initial concentration in receiving fluid at the first sample, $\mathrm{Vr}$ and $\mathrm{Vs}$ are volumes of the receptor chamber, ie $6.4 \mathrm{~mL}$, and volume of sample withdrawn $(500 \mu \mathrm{L})$. Similarly, $\mathrm{A}$ is the area of the diffusion cell $\left(1.77 \mathrm{~cm}^{2}\right){ }^{52}$

Furthermore, flux is the amount of permeant crossing the membrane into the circulatory system per unit time. The flux $\left(\mu \mathrm{g} / \mathrm{cm}^{2} / \mathrm{h}\right)$ was calculated from the slope of the linear portion of the cumulative quantity of drug permeated per unit area versus time plot.

\section{Skin Retention Studies}

After performing the permeation studies, skin samples were removed from the FDC and washed with $7.4 \mathrm{pH}$ phosphate buffer solution to remove surface adhered drug formulation. Moreover, washed skin samples were dried with tissue paper and cut into small pieces with the help of a surgical scissor. The small skin pieces were placed into a specified quantity of methanolic buffer solution (methanol 2:10 phosphate buffer). Furthermore, the methanolic buffer solution medium containing skin samples was subjected to homogenization in an Ultra-Turrax homogenizer (T45DX; IKAWerk, Germany) at 10,000 psi for $2 \mathrm{~min}$. All samples were filtered through a $0.45 \mu \mathrm{m}$ syringe filter (Millipore) and were analyzed using HPLC. All the experiments were performed in triplicate. ${ }^{53}$

\section{HPLC Analysis}

Samples obtained from ex vivo studies were analyzed using HPLC (Shimadzu, Japan), equipped with a pump (SPD-20A) and UV-detector (LC-20AT) at an oven temperature of $30{ }^{\circ} \mathrm{C}$. The separation of all the samples was done using a C18 column (Discovery ${ }^{\circledR}$, Cat\# $504,971)$ having a specification of $250 * 4.6,5 \mu \mathrm{m}$. The mobile phase was filtered through a nylon filter membrane $(0.45 \mu \mathrm{m})$ and degassed over an ultrasonicator for $10 \mathrm{~min}$. The mobile phase constituted acetonitrile and water in a ratio of 80:20 v/v. All samples were analyzed at $210 \mathrm{~nm}$ through a UV detector and the peaks were automatically recorded in the software. The flow rate was $1 \mathrm{~mL} / \mathrm{min}$ and the injection volume was $20 \mu \mathrm{L}$. The retention time recorded for finasteride was $2.5 \mathrm{~min}$.

The method validation was performed for precision, accuracy and specificity following international guidelines. However, this method was specific because no interference was observed between the skin and all subjected samples of formulations. The accuracy and precision of the system were $92 \%$ to $96 \%$, respectively.

\section{Statistical Analysis}

Data were analyzed using GraphPad Prism version 8.0.1 (244) software. Results with the corresponding standard deviation were expressed as a mean of at least three experiments. Statistical data analysis was conducted by employing one-way analysis of variance (ANOVA) and the statistically significant difference was denoted by 
$p<0.05$. Where appropriate, Student's $t$-test $p<0.05$ was used.

\section{Results and Discussion}

\section{Solubility Determination}

The selection of the oil and surfactants used for the formation of nanosystems was based on measurements of solubility as shown in Figure 1. The solubility of finasteride was determined in three different oils, ie oleic acid, eucalyptus and olive oils, as well as in two surfactants, ie Span 80 and Tween 80 . The FNS was highly soluble in oleic acid $(50.41 \pm 2.5 \mathrm{mg} / \mathrm{mL})$ as compared to eucalyptus $(40.03 \pm 4.4 \mathrm{mg} / \mathrm{mL})$ and olive oil $(38.55 \pm 2.5 \mathrm{mg} / \mathrm{mL})$. However, the solubility of FNS was significantly different among oleic acid and olive oil (Student's $t$-test: $p=0.01$ ) as mentioned for other BCS class II drugs. ${ }^{54}$ Oleic acid samples appeared clear homogeneous with no traces of turbidity and phase separation. Whereas eucalyptus and olive oil solutions with the drug were turbid in nature with clear phase separation. Similarly, FNS has good solubility in both surfactants and no significant difference was observed, ie Tween 80 (37.06 $\pm 2.56 \mathrm{mg} / \mathrm{mL}$ ), Span 80 (43 $\pm 2.1 \mathrm{mg} / \mathrm{mL}$ ) (Student's $t$-test: $p>0.05$ ). It was reported in previous studies that FNS showed more than $30 \mathrm{mg} / \mathrm{mL}$ solubility in Tween $80 .{ }^{55}$

\section{Formulation of NSs}

A stable, translucent and milky blank NS (F0) was first developed that was loaded with drug and further decorated

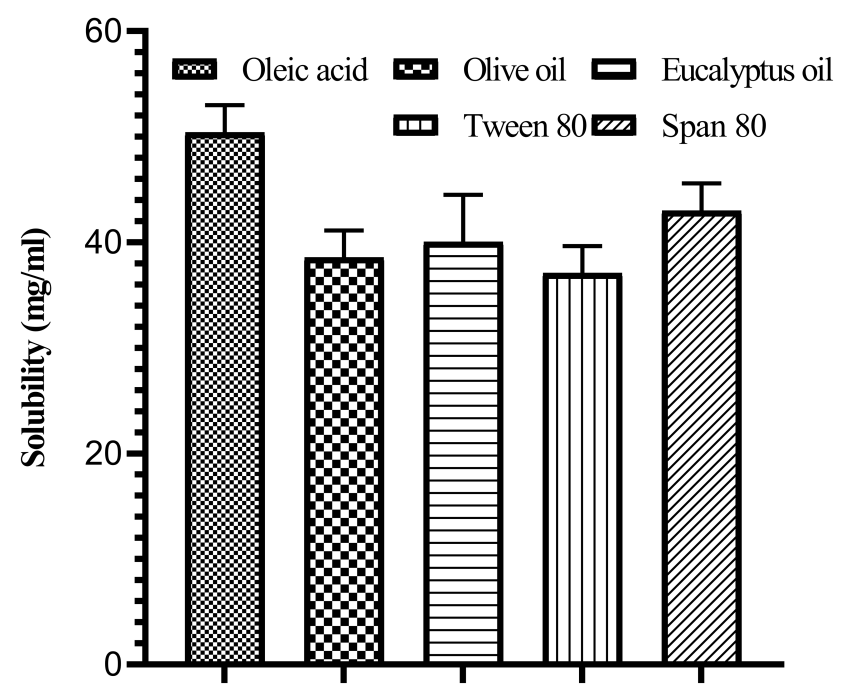

Figure I Solubility of finasteride in oils and emulgents. Experiments were carried out in triplicate $(n=3)$. Error bar shows standard deviation. Level of significance was determined using Student's $t$-test $(p<0.05)$. with chitosan (F1-F5; Table 1). Whereas FNS-NSs (F1-F5) are translucent but are milky yellowish colored. Because of the high disruptive forces generated in the abovementioned method, the oil and aqueous phases of the NS were forced to intersperse and substantially form a nanosized particulate system. The oleic acid (fatty acid) was used as a penetration enhancer which acts on the stratum corneum, ${ }^{56}$ causing delipidization and dekeratinization of the phospholipid membrane and enhanced penetration of the drug. The nonionic surfactants (Span 80 and Tween 80) were selected due to their known safety and biocompatibility, which are less affected by $\mathrm{pH}$ and changes in ionic strengths during preparation of NSs. ${ }^{57}$ They helped to reduce the interfacial tension between droplets and form a stable emulsion with minimal skin irritancy. ${ }^{58}$ According to the protocol, oil/ water NSs were developed by the addition of the oil phase into the water phase followed by high-pressure homogenization. Moreover, all the developed formulations were stored at room temperature for 28 days in order to be used for stability studies. There was no sign of sedimentation, change in color and phase separation after daily visual inspection up to the specified time. The rationale for selecting the topical nanosystem was to incorporate lipophilic and hydrophilic constituents in a nanosystem. Interestingly, FNS has a lipophilic nature, therefore, oil and the surfactants help to make isotropic NS, which could enhance the solubilization of molecules. Similarly, chitosan was used in the surface coating of nanoparticles to improve their biocompatibility and targetability. ${ }^{59}$ Chitosan has a wide range of applications in the drug delivery because of aqueous medium solubility, cationic functionality and being a natural biodegradable polymer. ${ }^{60}$ Different concentrations of low molecular weight chitosan were used for the encapsulation of FNS-NSs. Chitosan interacts with biological fluids, due to its mucoadhesive properties, and thereby increases the retention of drug at the target site. Chitosan was found to be helpful in the development of a sustained release drug delivery system. ${ }^{61,62}$ Our developed NS formulations have shown similar characteristics as mentioned in a previously reported study, where chitosan coated naringenin depicted a sustained release pattern. ${ }^{63}$

\section{Drug Entrapment Efficiency}

FNS was effectively encapsulated in both chitosan decorated and undecorated NSs. The average entrapment efficiency of the undecorated NS (F1) was $69.44 \pm 2.2 \%$ $(n=3)$. Whereas, the FNS E.E tends to be higher with decorated FNS-NSs, ie $73.89 \pm 0.51 \%(\mathrm{n}=3)$ for $\mathrm{F} 2$, 
$74.27 \pm 3.5 \%(n=3)$ for $F 3,75.66 \pm 0.25 \%(n=3)$ for $F 4$ and $77.2 \pm 3.64 \%(n=3)$ respectively for F5. The experimental results revealed that chitosan improved the \% E.E of all developed FNS-NSs. It could be observed that the surface of the NSs could be decorated with chitosan, which prevented the leakage of drug from the nanocarrier. These observations are in accordance with the previous investigation, which demonstrated that by increasing the concentration of chitosan the \% E.E was also improved. ${ }^{64}$

\section{Thermodynamic Stability Studies}

A thermodynamically stable NS should not lose its stability when subjected to various environmental stress conditions. FNS-NSs were able to attain their stability in different environmental conditions and there was no observable sign of turbidity, color change, phase separation, creaming and particle aggregations. In sequence, centripetal forces can facilitate phase separation due to variation in densities of oil and water. All the developed NSs were able to clear the centrifugation test, which indicates that the NS was homogeneous and stable. The emulsifying agents used in the formulations had a significant role in resisting phase separation and sedimentation during centrifugation tests. Furthermore, in the heating cycle, there was no sign of phase separation as heating may cause the melting of droplets that leads to phase separation. The above results indicate that the prepared NSs have a symmetric and organized structure and a thermodynamically stable system. The outcomes were consistent with preceding reports of a topical nanoemulsion gel of 5-fluorouracil. ${ }^{65}$

\section{Characterization of NSs}

The droplet size of the topical nanosystem has a remarkable role in drug absorption and bioavailability. The blank nanosystem (F0) had the smallest droplet size $(18 \mathrm{~nm}$, Student's $t$-test: $p<0.05$ ) and was statistically different from other FNS-NSs that was ascribed to the relatively higher quantity of water and absence of chitosan and drug. Similarly, after drug loading in NS (F1) the drug either adsorbed at the surface or was entrapped into the inner core of the NS, which could have significantly increased in the size of the droplet as compared to F0 (Student's $t$-test: $p=0.008$ ). It had been hypothesized that apart from the microstructure of the NSs, the drug could have also interacted with excipients used in the formulation and the resultant particle size was increased. Moreover, due to the employment of chitosan in increasing concentration, the droplet size FNS-NSs (F2-F5) were also significantly increased (Student's $t$-test: $p<0.05$ ), thus endorsing deposition of a thick coat of chitosan on the surface of FNS-NSs as shown in Table 2. Substantially, our droplet size results show a close resemblance with earlier studies that chitosan decorated nanoemulsions had larger particle size as compared to undecorated nanoemulsions. ${ }^{25,66}$

The PDI is used as an index to determine particle homogeneity. Formulations consisting of homogeneous and uniformly distributed particles would give lower PDI values as a measure of uniform particle distribution. To avoid batch to batch variation, keeping the rate of drug release steady and controllable is important to attain a minimum PDI. As mentioned in Table 2, the PDI values ranged from $0.329 \pm 0.02$ to $0.278 \pm 0.07$ for all NS compositions indicating a relatively small distribution of particle size, ${ }^{67}$ suitability of provided conditions and quantities of ingredients to achieve a monodispersed particle. Additionally, surface charge values of both blank (F0) and undecorated FNS-NS (F1) were in the range of $-10.16 \mathrm{mV}$ and $-12.89 \mathrm{mV}$. And chitosan decorated FNS-NSs bear high positive surface charge with values in the range of +56.1 to $+61.65 \mathrm{mV}$. The cationic charge was probably due to the presence of cationic polymer (chitosan) over the surface of the NSs. The chitosan decoration of FNSNSs resulted in both an increase in droplet size and

Table 2 Physicochemical Characteristics of Finasteride Nanosystems

\begin{tabular}{|l|c|c|c|c|c|}
\hline Formulation & Droplet Size (nm) & Size Distribution (PDI) & Surface Charge (mV) & pH & Specific Viscosity \\
\hline F0 & $18 \pm 0.2$ & $0.329 \pm 0.02$ & $-10.16 \pm 0.002$ & $5.4 \pm 0.06$ & $0.002 \pm 0.06$ \\
FI & $41.6 \pm 0.4$ & $0.480 \pm 0.009$ & $-12.89 \pm 0.226$ & $5.1 \pm 0.05$ & $0.009 \pm 0.01$ \\
F2 & $242 \pm 8.42$ & $0.316 \pm 0.05$ & $56.35 \pm 0.63$ & $5.5 \pm 0.07$ & $0.266 \pm 0.02$ \\
F3 & $344 \pm 1.41$ & $0.269 \pm 0.006$ & $59.50 \pm 1.27$ & $5.6 \pm 0.03$ & $0.723 \pm 0.01$ \\
F4 & $512 \pm 44.54$ & $0.338 \pm 0.03$ & $61.65 \pm 1.48$ & $5.4 \pm 0.09$ & $6.628 \pm 0.06$ \\
F5 & $864 \pm 82.23$ & $0.278 \pm 0.07$ & $56.10 \pm 0.00$ & $5.5 \pm 0.02$ & $22.73 \pm 0.02$ \\
\hline
\end{tabular}

All the experiments were done in triplicate $(n=3)$. Level of significance was determined by employing Student's t-test $(p<0.05)$. 
higher zeta potential values which is an indicator of better stability of dispersed particles due to stronger intermolecular electrostatic repulsion among molecules.68,69 The shift in zeta potential from negative to positive charge suggests the surface decoration of the FNS-NSs with chitosan. Chitosan with a higher degree of deacetylation results in higher zeta potential due to higher load density of more deacetylated chitosan. ${ }^{70}$ In the same way, interactions of both positive and negative charged NSs to the skin are a complex process. However, the skin tends to attract both the same as well as opposite charge particles. Although, positive surface charged particles interact more with the skin.71,72 The morphological analysis of all developed NSs is given in Figure 2. The TEM images indicated the formation of tiny, discrete monodispersed and spherical shaped NSs. The TEM analysis also confirms our data from dynamic light scattering, where incorporating different concentration of chitosan significantly increased the structural size of NSs but overall morphological features were not influenced by increasing chitosan concentration. $^{73}$
The $\mathrm{pH}$ values obtained from all developed NSs were between $5.4 \pm 0.06$ and $5.5 \pm 0.02$ respectively. The surface of the skin is protected by a hydrolipid film which is slightly acidic that solubilizes the sebaceous gland secretion and can also prevent water from evaporation, thereby helping to keep the skin hydrated. The values obtained in this assay were consistent with the $\mathrm{pH}$ guidelines for topical formulations which should have a $\mathrm{pH}$ value of 56 , close to the $\mathrm{pH}$ of the stratum corneum and thus avoid skin irritation. ${ }^{74,75}$ Additionally, F0 and F1 were less viscous $(0.002 \pm 0.06$ and $0.009 \pm 0.01)$ as compared to other FNS-NSs. The higher viscosity of F2 to F5 $(0.266 \pm 0.02$ to $22.73 \pm 0.02$ ) was believed to be associated with the presence of low molecular weight chitosan. So, the chitosan decorated FNS-NSs could be easily applied and removed from the skin surface as compared to F1. The $\mathrm{pH}$ and specific viscosity values are depicted in Table 2 .

\section{In vitro Release Studies}

Overall, the developed finasteride NSs exhibited sustained drug release patterns in simulated skin physiological conditions. The in vitro drug release behavior from F1 to F5 is
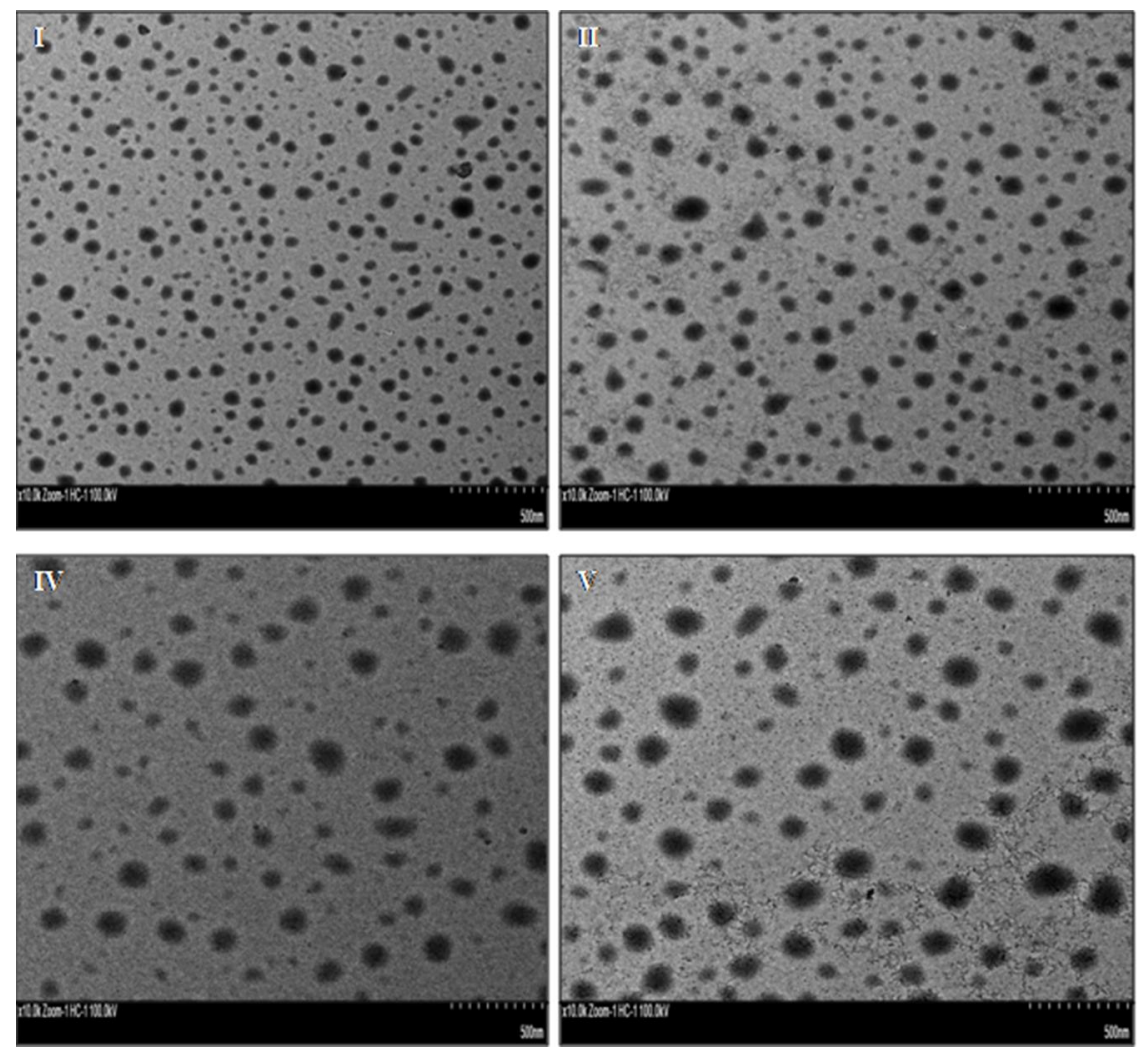
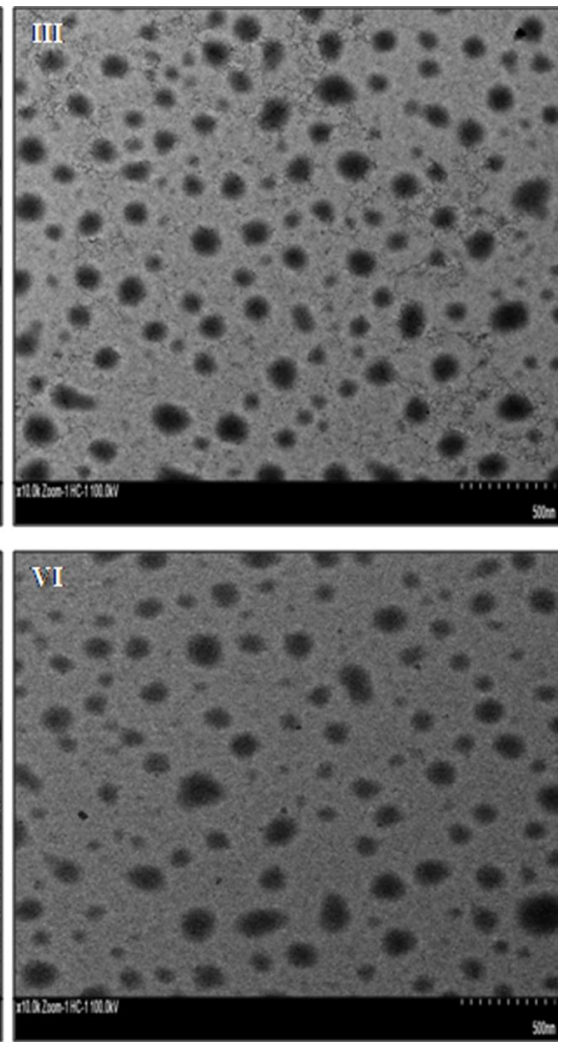

Figure 2 TEM images of nanosystems. (I) Blank nanosystem (F0). (II) Undecorated finasteride nanosystem (FNS-NS) (FI). (III) $0.125 \%$ FNS-NS (F2). (IV) 0.25\% FNS-NS (F3). (V) $0.5 \%$ FNS-NS (F4). (VI) $1 \%$ FNS-NS (F5). 


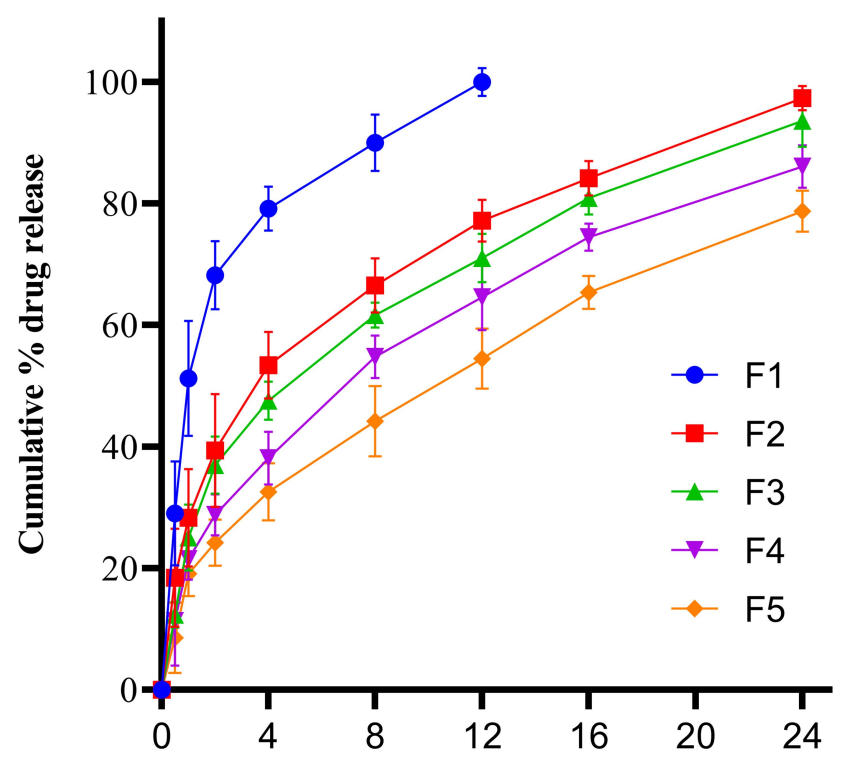

Time (hr)

Figure 3 In vitro release profile of finasteride nanosystems. Experiments were performed in triplicate $(n=3)$. Error bar shows standard deviation. Level of significance was determined by one-way ANOVA $(p<0.05)$.

shown in Figure 3. All chitosan decorated FNS-NSs exhibited an extended-release pattern with no significant variation as compared to F1 (ANOVA, $p=0.33$; Table 4). The F1 showed a burst release pattern because of its smaller droplet size that provided a greater surface area for dissolution, therefore releasing the drug within $12 \mathrm{~h}$. The F2 and F3 samples comprising $0.125 \%$ and $0.25 \%$ chitosan showed a sustained release pattern irrespective of the chitosan concentration in the formulations $(97 \%$ and $93 \%$ respectively in $24 \mathrm{~h}$ ). But, due to the larger particle size and high viscosity, both F4 and F5 chitosan-coated formulations showed very slow release patterns and approximately $80 \%$ of FNS was released after the specified time. However, chitosan decoration could influence the method of drug release but drug release does not change. In addition, investigations had revealed that variations in the concentration of chitosan $(0.125 \%$ to $1 \%)$ could indeed influence drug release behavior by making drug release in a prolonged manner compared to the undecorated formulation. ${ }^{64}$ The same pattern of drug release was observed in our chitosan decorated FNS-NSs.

\section{Drug Release Kinetics}

The findings obtained from the release analysis of our developed formulations fitted to different kinetic models of drug release are summarized in Table 3 . The best model for each formulation was chosen on the basis of a regression coefficient $\left(R^{2}\right)$ value close to 1 . The first-order $R^{2}$ value was higher than that of zero-order for all formulations, indicating concentration-dependent release of the drug. The $\mathrm{R}^{2}$ value of the Higuchi model was higher than 0.5 , which suggests that the drug was released following the diffusion process while the Korsmeyer-Peppas "N" value was higher than 0.5 for all formulations indicating a non-Fickian or anomalous mechanism.

\section{Ex vivo Permeation and Drug Retention Studies}

The permeation studies were carried out for all formulations (F1-F5) each comprising an equal amount of drug $(0.05 \% \mathrm{w} / \mathrm{w})$, oil and surfactants. However, the formulations from F2 to F5 were having different concentrations of chitosan. The sustained release of FNS in the in vitro drug release analysis facilitates the delayed release of FNS into the skin layers. In addition, chitosan exhibits mucoadhesive properties that favor skin adhesive strength and increase the time of drug residence in the skin. Statistically, it was found that higher drug diffusion occurs from the undecorated formulation (F1) as compared to other formulations (F2-F5) that have a lesser permeation rate across rat skin (ANOVA, $p=0.01$ ). The results are shown in Figure 4.

There was a maximum drug permeation from $\mathrm{F} 1$ (ie $45.18 \mu \mathrm{g} / \mathrm{cm}^{2}$ ) that associated with its anionic surface charge and our findings were consistent with previous

Table 3 Drug Release Kinetics

\begin{tabular}{|l|c|c|c|c|c|c|}
\hline Formulation & Zero Order $\mathbf{( R}^{\mathbf{2})}$ & First Order $\left.\mathbf{( R}^{\mathbf{2}}\right)$ & Higuchi Model $\left.\mathbf{( R}^{\mathbf{2}}\right)$ & \multicolumn{2}{|c|}{$\begin{array}{c}\text { Korsmeyer-Peppas } \\
\left(\mathbf{R}^{\mathbf{2}} \mathbf{( \mathbf { N } )}\right.\end{array}$} & Mechanism of Drug Release \\
\hline FI & 0.652 & 0.959 & 0.862 & 0.833 & 0.606 & Non-Fickian diffusion \\
F2 & 0.836 & 0.964 & 0.978 & 0.961 & 0.699 & Non-Fickian diffusion \\
F3 & 0.881 & 0.984 & 0.980 & 0.979 & 0.808 & Non-Fickian diffusion \\
F4 & 0.913 & 0.988 & 0.990 & 0.948 & 0.725 & Non-Fickian diffusion \\
F5 & 0.909 & 0.968 & 0.974 & 0.968 & 0.731 & Non-Fickian diffusion \\
\hline
\end{tabular}




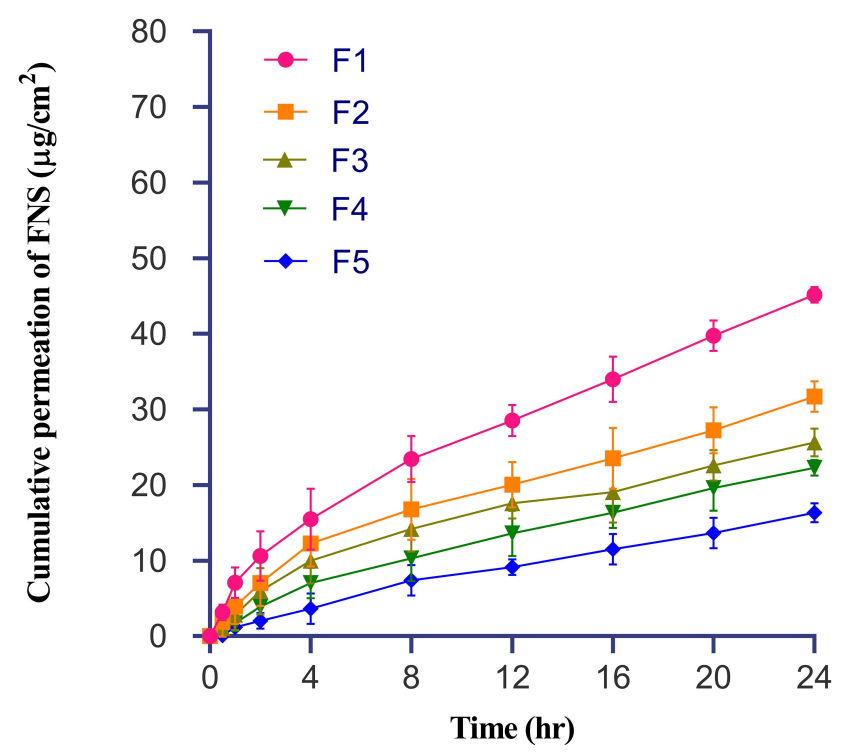

Figure 4 Ex vivo permeation of finasteride through rat skin. Experiments were performed in triplicate $(n=3)$. Error bar shows standard deviation. Level of significance was determined by one-way ANOVA $(p<0.05)$.

investigations which reported that anionic charge nanovesicles depicted a higher permeation rate by the accumulation of drugs when interacted with skin than cationic charge particles. ${ }^{22,76-78}$ This high permeability of F1 may be due to low viscosity, smaller globular size and spherical shape, and thus could be easily squeezed from the skin. Small nanoparticles that establish close contact with the skin provide a larger area for drug permeation and release a high concentration of drugs. Hence, the drug from the F1 formulation easily penetrated the skin layers and experimental analysis revealed that a smaller globular size nanoparticulate system exhibited a higher drug permeability rate. $^{79,80}$ Moreover, flux was also higher in F1 as compared to chitosan decorated formulations, as results are described in Table 4. In addition, due to the external water phase of the FNS-NSs, hydration of the stratum corneum allows the corneum cells to swell, thereby making the channels for drug passage wider, resulting in an increased lipophilic drug diffusivity. ${ }^{81}$ Moreover, the presence of oleic acid can generate pores on corneocytes of the uppermost skin layer, thus changing the resistance of the stratum corneum towards hydrophobic drug diffusion. Whereas the suitable amount of surfactants can alter SC barrier functions by penetrating the drug from the epidermis to the dermis layer. ${ }^{82}$

The extent of drug permeation from chitosan decorated FNS-NSs follows descending order as compared to F1 after $24 \mathrm{~h}$, as data ascribed in Table 4. It was noteworthy that a decrease in the permeation and flux rate was interlinked with chitosan concentration in the NSs. Furthermore, particle size and charge have a greater impact on the permeation profile of the drug across the skin. Although, the skin has negative surface charge and thus attracts positive charge with more force as compared to negative charged NS. But chitosan decorated formulations (F2-F5) have low permeability and lower flux rate than F1, which could be attributed to their larger particle size and higher viscous nature that allows the drug permeation in a sustained manner through the skin membrane. Recently, a study was conducted that supports our finding that a larger particle size of NSs depicted lower drug permeation. $^{83}$

After $24 \mathrm{~h}$ of permeation studies, the drug skin retention was assessed by chromatographic technique, as drugs need to reside in the skin for a longer duration to elicit their therapeutic action when topically applied. The results of drug skin retention were in descending order F5 $>$ F4 $>$ F3 $>$ F2 $>$ F1 (Table 4). As compared to the F1 formulation, the chitosan decorated FNS-NSs (F2-F5) were able to retain significantly higher finasteride fraction in the skin (Student's $t$-test: $p<0.05$ ) as shown in Figure 5. The direct cause behind higher retention of FNS-NSs can be attributed to chitosan in the optimized formulations. This increase in FNS retention might be due to the interaction between cationic charged chitosan

Table 4 Finasteride Nanosystem Patterns of Drug Release, Permeation, Flux and Drug Retention

\begin{tabular}{|l|c|c|c|c|}
\hline Formulation & $\begin{array}{c}\text { Cumulative Drug Release } \\
\text { (\%) }\end{array}$ & $\begin{array}{c}\text { Cumulative Drug Permeation } \mathbf{( \mu g /} \\
\left.\mathbf{c m}^{\mathbf{2}}\right)\end{array}$ & $\begin{array}{c}\text { Flux }\left(\boldsymbol{\mu g} / \mathbf{c m}^{\mathbf{2}} \mathbf{\text { I }}\right. \\
\mathbf{h})\end{array}$ & $\begin{array}{c}\text { Average Drug Retention } \\
\text { (\%) }\end{array}$ \\
\hline FI & $100 \pm 2.28$ & $45.18 \pm 1.03$ & $1.34 \pm 0.005$ & $6.38 \pm 0.18$ \\
F2 & $97.31 \pm 2.03$ & $31.70 \pm 2.01$ & $0.89 \pm 0.015$ & $6.89 \pm 0.32$ \\
F3 & $93.62 \pm 4.26$ & $25.65 \pm 1.84$ & $0.74 \pm 0.01$ & $8.17 \pm 0.06$ \\
F4 & $86.08 \pm 3.49$ & $22.26 \pm 1.06$ & $0.70 \pm 0.02$ & $8.45 \pm 1.04$ \\
F5 & $78.73 \pm 3.35$ & $16.35 \pm 1.25$ & $0.59 \pm 0.026$ & $10.81 \pm 0.37$ \\
\hline
\end{tabular}

Analyses were carried out in triplicate $(n=3 \pm S D)$. 


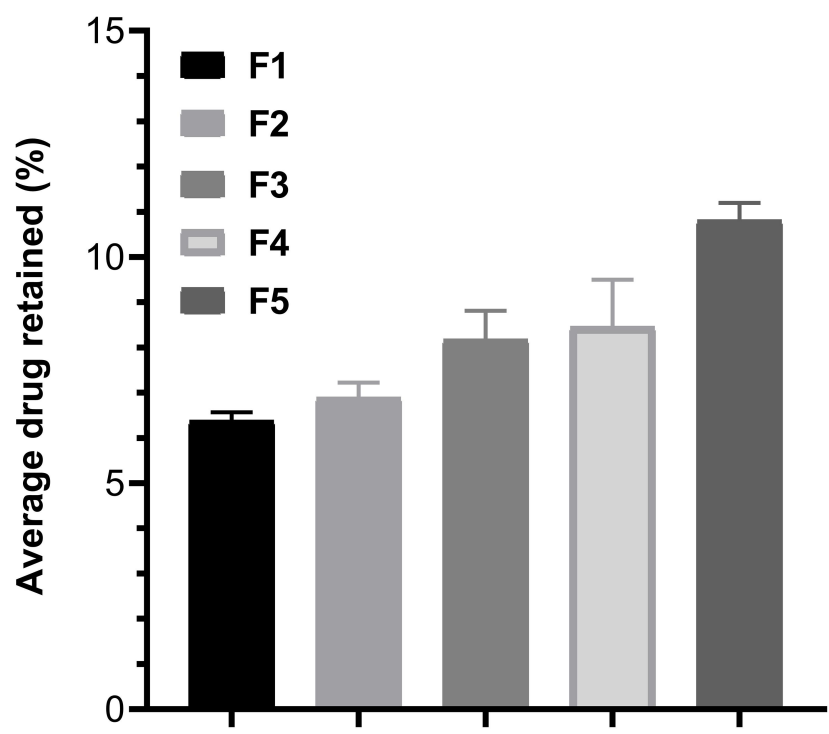

Figure 5 Finasteride skin deposition profile. Experiments was performed in triplicate $(n=3)$. Error bar shows standard deviation. Level of significance was determined using Student's $t$-test $(p<0.05)$.

and the hydrophobic FNS moiety with anionic charged lipid skin membrane. ${ }^{84}$ Based on these findings, it can be inferred that F5 has a higher skin affinity. For this purpose, chitosan can be used in topical formulations to hold the formulation in both the epidermal and the dermal layers of the skin. A higher fraction of the particulate matter was identified from F5 as compared to other FNSNSs. These results could be helpful in the development of a suitable formulation for the treatment of androgenic alopecia. Moreover, when comparing the rate of drug deposition of each formulation, it was envisaged that the use of chitosan in the formulations employed exhibited a higher accumulation of finasteride in the skin. Our findings were consistent with other studies where, after incorporation of chitosan, the drug showed a substantially higher retention rate in the skin. ${ }^{18}$

\section{Conclusion}

Our developed nanosystems that comprised the same organic and inorganic materials, with and with or without polymeric decoration, played a critical role in achieving the delivery of FNS into or across the skin. In this study, we successfully developed and characterized oil/water FNS-NSs for skin delivery. In our findings, the permeation of undecorated FNS-NS was higher as compared to chitosan decorated NSs. Moreover, the drug retention was remarkably higher when the NSs were decorated with chitosan. In conclusion, our formulation of $1 \%$ chitosan
(F5) depicted a better retention rate of the drug in skin as compared to other formulations and thus was deemed a promising vesicle for topical delivery in the possible treatment of androgenic alopecia and could become a potential alternative for oral intake of FNS, overcoming the related unwanted harmful side effects.

\section{Disclosure}

The authors report no conflict of interest in this work.

\section{References}

1. Santos Z, Avci P, Hamblin MR. Drug discovery for alopecia: gone today, hair tomorrow. Expert Opin Drug Discov. 2015;10(3):269292. doi:10.1517/17460441.2015.1009892

2. Upadhyay DK, Sharma A, Kaur N, Das GG, Narang RK. Nanoemulgel for efficient topical delivery of finasteride against androgenic alopecia. J Pharm Innov. 2020. doi:10.1007/s12247020-09483-9

3. Dhurat R, Sharma A, Rudnicka L, Kroumpouzos G, Kassir M, Galadari H. 5-Alpha reductase inhibitors in androgenetic alopecia: shifting paradigms, current concepts, comparative efficacy, and safety. Clin Drug Investig. 2020;(December 2019):1-5.

4. Shin YS, Karna KK, Choi BR, Park JK. Finasteride and erectile dysfunction in patients with benign prostatic hyperplasia or male androgenetic alopecia. World J Mens Health. 2019;37(2):157-165. doi:10.5534/wjmh. 180029

5. Ahmed TA, El-Say KM, Hosny KM, Aljaeid BM. Development of optimized self-nanoemulsifying lyophilized tablets (SNELTs) to improve finasteride clinical pharmacokinetic behavior. Drug Dev Ind Pharm. 2018;44(4):652-661. doi:10.1080/ 03639045.2017.1405977

6. Seo JO, Yumnam S, Jeong KW, Kim SY. Finasteride inhibits melanogenesis through regulation of the adenylate cyclase in melanocytes and melanoma cells. Arch Pharm Res. 2018;41(3):324-332. doi:10.1007/s12272-018-1002-x

7. Mady FM, Aly UF. Experimental, molecular docking investigations and bioavailability study on the inclusion complexes of finasteride and cyclodextrins. Drug Des Devel Ther. 2017;11:1681-1692. doi:10.2147/DDDT.S135084

8. Xiao Q, Wang L, Supekar S, et al. Structure of human steroid $5 \alpha-$ reductase 2 with anti-androgen drug finasteride. Res Sq. 2020;1-23.

9. Azeem A, Khan ZI, Aqil M, Ahmad FJ, Khar RK, Talegaonkar S. Microemulsions as a surrogate carrier for dermal drug delivery. Drug Dev Ind Pharm. 2009;35(5):525-547. doi:10.1080/ 03639040802448646

10. Afiune LAF, Ushirobira CY, Barbosa DPP, et al. Novel iron oxide nanocarriers loading finasteride or dutasteride: enhanced skin penetration for topical treatment of alopecia. Int J Pharm. 2020;587 (May):119709. doi:10.1016/j.ijpharm.2020.119709

11. Diviccaro S, Melcangi RC, Giatti S. Post-finasteride syndrome: an emerging clinical problem. Neurobiol Stress. 2020;12(October 2019):100209. doi:10.1016/j.ynstr.2019.100209

12. Rowland D, Motofei I, Păunică I, et al. Androgenic alopecia; the risk-benefit ratio of finasteride. J Mind Med Sci. 2018;5(1):1-6. doi:10.22543/7674.51.P16

13. Paunica S, Giurgiu M, Vasilache A. Finasteride adverse effects and post-finasteride syndrome; implications for dentists. J Mind Med Sci. 2016;3(1).

14. González-Louzao R, Lucas-Abellán $\mathrm{C}$, Pérez-Sánchez $\mathrm{H}$, et al. Encapsulation of finasteride with native and modified $\gamma$-cyclodextrins. Extensive characterization of the complexes. Int $J$ Pharm. 2020;587(july):119619. doi:10.1016/j.ijpharm.2020.119619 
15. Soleymani SM, Salimi A. Enhancement of dermal delivery of finasteride using microemulsion systems. Adv Pharm Bull. 2019;9 (4):584-592. doi:10.15171/apb.2019.067

16. Maranduca M, Hurjui L, Branisteanu D, et al. Skin - a vast organ with immunological function (review). Exp Ther Med. 2020;18-23.

17. Rabiei M, Kashanian S, Samavati SS, Jamasb S, McInnes SJP. Nanomaterial and advanced technologies in transdermal drug delivery. $J$ Drug Target. 2020;28(4):356-367. doi:10.1080/ 1061186X.2019.1693579

18. Caon T, Porto LC, Granada A, et al. Chitosan-decorated polystyreneb-poly(acrylic acid) polymersomes as novel carriers for topical delivery of finasteride. Eur $J$ Pharm Sci. 2014;52(1):165-172. doi:10.1016/j.ejps.2013.11.008

19. Rao Y, Zheng F, Liang X, Wang H, Zhang J, Lu X. Penetration profile and human cadaver skin distribution of finasteride from vesicular nanocarriers. Drug Deliv. 2013;22(8):1003-1009. doi:10.3109/ 10717544.2013.839128

20. Madheswaran T, Baskaran R, Yong CS, Yoo BK. Enhanced topical delivery of finasteride using glyceryl monooleate-based liquid crystalline nanoparticles stabilized by cremophor surfactants. Am Assoc Pharm Sci. 2014;15(1):44-51.

21. Caserini M, Radicioni M, Leuratti C, Terragni E, Iorizzo M, Palmieri R. Effects of a novel finasteride $0.25 \%$ topical solution on scalp and serum dihydrotestosterone in healthy men with androgenetic alopecia. Int J Clin Pharmacol Ther. 2016;54(1):19-27. doi:10.5414/ CP202467

22. Roque LV, Dias IS, Cruz N, et al. Design of finasteride-loaded nanoparticles for potential treatment of alopecia. Skin Pharmacol Physiol. 2017;30(4):197-204. doi:10.1159/000475473

23. Lima SGB, Pinho LAG, Pereira MN, et al. Preformulation studies of finasteride to design matrix systems for topical delivery. $J$ Pharm Biomed Anal. 2018;161:273-279. doi:10.1016/j.jpba.2018.08.056

24. Khan MZU, Khan SA, Ubaid M, Shah A, Kousar R, Murtaza G. Finasteride topical delivery systems for androgenetic alopecia. Curr Drug Deliv. 2018;15(8):1100-1111. doi:10.2174/ 1567201815666180124112905

25. Luesakul U, Puthong S, Sansanaphongpricha K, Muangsin N. Quaternized chitosan-coated nanoemulsions: a novel platform for improving the stability, anti-inflammatory, anti-cancer and transdermal properties of plai extract. Carbohydr Polym. 2020;230 (November):115625. doi:10.1016/j.carbpol.2019.115625

26. Development L, Vitro I, Permeation S, Santos CM, Oliveira De RB, Arantes VT. Amphotericin B-loaded nanocarriers for topical treatment of cutaneous amphotericin b-loaded nanocarriers for topical treatment of cutaneous leishmaniasis: development, characterization, and in vitro skin permeation studies. J Biomed Nanotechnol. 2012.

27. Nikolic I, Jasmin Lunter D, Randjelovic D, et al. Curcumin-loaded low-energy nanoemulsions as a prototype of multifunctional vehicles for different administration routes: physicochemical and in vitro peculiarities important for dermal application. Int $J$ Pharm. 2018;550(1-2):333-346. doi:10.1016/j.ijpharm.2018.08.060

28. Pucek A, Tokarek B, Waglewska E, Bazylińska U. Recent advances in the structural design of photosensitive agent formulations using "soft" colloidal nanocarriers. Pharmaceutics. 2020;12(6):1-33. doi:10.3390/pharmaceutics 12060587

29. Bazylińska U, Saczko J. Nanoemulsion-templated polylelectrolyte multifunctional nanocapsules for DNA entrapment and bioimaging. Colloids Surf B Biointerfaces. 2016;137:191-202. doi:10.1016/j. colsurfb.2015.07.056

30. Naseema A, Kovooru L, Behera AK, Kumar KPP, Srivastava P. A critical review of synthesis procedures, applications and future potential of nanoemulsions. Adv Colloid Interface Sci. 2021;287:102318. doi:10.1016/j.cis.2020.102318

31. Paquin F, Rivnay J, Salleo A, Stingelin N, Silva C. Multi-phase semicrystalline microstructures drive exciton dissociation in neat plastic semiconductors. J Mater Chem C. 2015;3:10715-10722.
32. Hu R, Zheng H, Cao J, Davoudi Z, Wang Q. Synthesis and in vitro characterization of carboxymethyl chitosan-CBA-doxorubicin conjugate nanoparticles as $\mathrm{pH}$-sensitive drug delivery systems. $J$ Biomed Nanotechnol. 2017;13(9):1097-1105. doi:10.1166/ jbn.2017.2407

33. Poovaiah N, Davoudi Z, Peng H, et al. Treatment of neurodegenerative disorders through the blood-brain barrier using nanocarriers. Nanoscale. 2018;10(36):16962-16983. doi:10.1039/C8NR04073G

34. Carter P, Narasimhan B, Wang Q. Biocompatible nanoparticles and vesicular systems in transdermal drug delivery for various skin diseases. Int $J$ Pharm. 2019;555:49-62. doi:10.1016/j. ijpharm.2018.11.032

35. Yang B, Jiang J, Jiang L, et al. Chitosan mediated solid lipid nanoparticles for enhanced liver delivery of zedoary turmeric oil in vivo. Int $J$ Biol Macromol. 2020;149:108-115. doi:10.1016/j. ijbiomac.2020.01.222

36. Wei X, Liao J, Davoudi Z, et al. Folate receptor-targeted and gshresponsive carboxymethyl chitosan nanoparticles containing covalently entrapped 6-mercaptopurine for enhanced intracellular drug delivery in leukemia. Mar Drugs. 2018;16(11):439. doi:10.3390/ md16110439

37. Vecchione R, Ciotola U, Sagliano A, Bianchini P, Diaspro A, Netti PA. Tunable stability of monodisperse secondary $\mathrm{O} / \mathrm{W}$ nano-emulsions. Nanoscale. 2014;6(15):9300-9307. doi:10.1039/C4NR02273D

38. McClements DJ, Gumus CE. Natural emulsifiers - biosurfactants, phospholipids, biopolymers, and colloidal particles: molecular and physicochemical basis of functional performance. Adv Colloid Interface Sci. 2016;234:3-26. doi:10.1016/j.cis.2016.03.002

39. Bonferoni MC, Sandri G, Rossi S, et al. A novel ionic amphiphilic chitosan derivative as a stabilizer of nanoemulsions: improvement of antimicrobial activity of cymbopogon citratus essential oil. Colloids Surf $\quad B \quad$ Biointerfaces. 2017;152:385-392. doi:10.1016/j. colsurfb.2017.01.043

40. Baldrick P. The safety of chitosan as a pharmaceutical excipient. Regul Toxicol Pharmacol. 2010;56(3):290-299. doi:10.1016/j. yrtph.2009.09.015

41. Nawaz A, Wong TW. Chitosan-carboxymethyl-5-fluorouracil-folate conjugate particles: microwave modulated uptake by skin and melanoma cells. $J$ Invest Dermatol. 2018;138(11):2412-2422. doi:10.1016/j.jid.2018.04.037

42. Bakshi P, Jiang Y, Nakata T, Akaki J, Matsuoka N, Banga AK. Formulation development and characterization of nanoemulsionbased formulation for topical delivery of heparinoid. J Pharm Sci. 2018;107(11):2883-2890. doi:10.1016/j.xphs.2018.07.015

43. Loureiro Contente DM, Pereira RR, Rodrigues AMC, da Silva EO, Ribeiro-Costa RM, Carrera Silva-Júnior JO. Nanoemulsions of acai oil: physicochemical characterization for the topical delivery of antifungal drugs. Chem Eng Technol. 2020;43(7):1424-1432. doi:10.1002/ceat.201900627

44. Rajitha P, Shammika P, Aiswarya S, Gopikrishnan A, Jayakumar R, Sabitha M. Chaulmoogra oil based methotrexate loaded topical nanoemulsion for the treatment of psoriasis. J Drug Deliv Sci Technol. 2019;49(October 2018):463-476. doi:10.1016/j. jddst.2018.12.020

45. Algahtani MS, Ahmad MZ, Ahmad J. Nanoemulsion loaded polymeric hydrogel for topical delivery of curcumin in psoriasis. J Drug Deliv Sci Technol. 2020;59:101847. doi:10.1016/j.jddst.2020.101847

46. Bazylińska U, Kulbacka J, Chodaczek G. Nanoemulsion structural design in co-encapsulation of hybrid multifunctional agents: influence of the smart PLGA polymers on the nanosystem-enhanced delivery and electro-photodynamic treatment. Pharmaceutics. 2019;11(8):405. doi:10.3390/pharmaceutics 11080405

47. Shah K, Chan LW, Wong TW. Critical physicochemical and biological attributes of nanoemulsions for pulmonary delivery of rifampicin by nebulization technique in tuberculosis treatment. Drug Deliv. 2017;24(1):1631-1647. doi:10.1080/10717544.2017.1384298 
48. Zhang Y, Zhang R, Illangakoon UE, et al. Copolymer composition and nanoparticle configuration enhance in vitro drug release behavior of poorly water-soluble progesterone for oral formulations. Int $J$ Nanomedicine. 2020;15:5389-5403. doi:10.2147/IJN.S257353

49. Khan NR, Wong TW. Microwave-aided skin drug penetration and retention of 5-fluorouracil-loaded ethosomes. Expert Opin Drug Deliv. 2016;13(9):1209-1219. doi:10.1080/17425247.2016.1193152

50. Salama A, Badran M, Elmowafy M, Soliman GM. Spironolactoneloaded leciplexes as potential topical delivery systems for female acne: in vitro appraisal and ex vivo skin permeability studies. Pharmaceutics. 2020;12(1):1-17.

51. Hussain A, Altamimi MA, Alshehri S, Imam SS, Shakeel F, Singh SK. Novel approach for transdermal delivery of rifampicin to induce synergistic antimycobacterial effects against cutaneous and systemic tuberculosis using a cationic nanoemulsion gel. Int J Nanomedicine. 2020;15:1073-1094. doi:10.2147/IJN.S236277

52. Lin HW, Xie QC, Huang X, et al. Increased skin permeation efficiency of imperatorin via charged ultradeformable lipid vesicles for transdermal delivery. Int $J$ Nanomedicine. 2018;13:831-842. doi:10.2147/IJN.S150086

53. Aragão Horoiwa T, Cortez M, Sauter IP, et al. Sugar-based colloidal nanocarriers for topical meglumine antimoniate application to cutaneous leishmaniasis treatment: ex vivo cutaneous retention and in vivo evaluation. Eur J Pharm Sci. 2020;147 (November2019):105295. doi:10.1016/j.ejps.2020.105295

54. Patel K, Sarma V, Vavia P. Design and evaluation of lumefantrine - oleic acid self nanoemulsifying ionic complex for enhanced dissolution. DARU J Pharm Sci. 2013;21(1). doi:10.1186/20082231-21-27

55. Fagir W, Hathout RM, Sammour OA, ElShafeey AH. Self-microemulsifying systems of finasteride with enhanced oral bioavailability: multivariate statistical evaluation, characterization, spray-drying and in vivo studies in human volunteers. Nanomedicine (Lond). 2015;10 (22):3373-3389. doi:10.2217/nnm.15.123

56. Sarheed O, Shouqair D, Ramesh K, Khaleel T, Boateng J, Drechsler M. Formation of stable nanoemulsions by ultrasound-assisted twostep emulsification process for topical drug delivery: effect of oil phase composition and surfactant concentration and loratadine as ripening inhibitor. Int J Pharm. 2019;576:118952. doi:10.1016/j. ijpharm.2019.118952

57. Chang Y, McClements DJ. Optimization of orange oil nanoemulsion formation by isothermal low-energy methods: influence of the oil phase, surfactant, and temperature. J Agric Food Chem. 2014;62 (10):2306-2312. doi:10.1021/jf500160y

58. Nastiti CMRR, Ponto T, Abd E, Grice JE, Benson HAE, Roberts MS. Topical nano and microemulsions for skin delivery. Pharmaceutics. 2017;9(4):1-25. doi:10.3390/pharmaceutics9040037

59. Ushirobira CY, Afiune LAF, Pereira MN, Cunha-Filho M, Gelfuso GM, Gratieri T. Dutasteride nanocapsules for hair follicle targeting: effect of chitosan-coating and physical stimulus. Int J Biol Macromol. 2020;151:56-61. doi:10.1016/j. ijbiomac.2020.02.143

60. Rizeq BR, Younes NN, Rasool K, Nasrallah GK. Synthesis, bioapplications, and toxicity evaluation of chitosan-based nanoparticles. Int J Mol Sci. 2019;20(22):22. doi:10.3390/ijms20225776

61. Parhi R. Drug delivery applications of chitin and chitosan: a review. Environ Chem Lett. 2020;18(3):577-594. doi:10.1007/s10311-02000963-5

62. Matos BN, Pereira MN. Chitosan nanoparticles loading oxaliplatin as a mucoadhesive topical treatment of oral tumors: iontophoresis further enhances drug delivery ex vivo. Int J Biol Macromol. 2020;154:1265-1275. doi:10.1016/j.ijbiomac.2019.11.001

63. Akrawi SH, Gorain B, Nair AB, et al. Development and optimization of naringenin-loaded chitosan-coated nanoemulsion for topical therapy in wound healing. Pharmaceutics. 2020;12(9):1-23.
64. Khalil M, Hashmi U, Riaz R, Rukh Abbas S. Chitosan coated liposomes (CCL) containing triamcinolone acetonide for sustained delivery: a potential topical treatment for posterior segment diseases. Int $J$ Biol Macromol. 2020;143(Ccl):483-491. doi:10.1016/j. ijbiomac.2019.10.256

65. Ahmad N, Ahmad R, Mohammed Buheazaha T, Salman AlHomoud H, Al-Nasif HA, Sarafroz M. A comparative ex vivo permeation evaluation of a novel 5-Fluorocuracil nanoemulsion-gel by topically applied in the different excised rat, goat, and cow skin. Saudi J Biol Sci. 2020;27(4):1024-1040. doi:10.1016/j.sjbs.2020.02.014

66. Abd-Allah H, Abdel-Aziz RTA, Nasr M. Chitosan nanoparticles making their way to clinical practice: a feasibility study on their topical use for acne treatment. Int J Biol Macromol. 2020;156:262270. doi:10.1016/j.ijbiomac.2020.04.040

67. Algharib SA, Dawood A, Zhou K, et al. Designing, structural determination and biological effects of rifaximin loaded chitosan- carboxymethyl chitosan nanogel. Carbohydr Polym. 2020;248 (April):116782. doi:10.1016/j.carbpol.2020.116782

68. Mady MM, Darwish MM. Effect of chitosan coating on the characteristics of DPPC liposomes. J Adv Res. 2010;1(3):187-191. doi:10.1016/j.jare.2010.05.008

69. Shao Y, Wu C, Wu T, et al. Eugenol-chitosan nanoemulsions by ultrasound-mediated emulsification: formulation, characterization and antimicrobial activity. Carbohydr Polym. 2018;193:144-152. doi:10.1016/j.carbpol.2018.03.101

70. Zafar S, Akhter S, Ahmad I, et al. Improved chemotherapeutic efficacy against resistant human breast cancer cells with co-delivery of docetaxel and thymoquinone by chitosan grafted lipid nanocapsules: formulation optimization, in vitro and in vivo studies. Colloids Surf B Biointerfaces. 2020;186(October):110603. doi:10.1016/j. colsurfb.2019.110603

71. Vogt A, Rancan F, Ahlberg S, et al. Interaction of dermatologically relevant nanoparticles with skin cells and skin. Beilstein $J$ Nanotechnol. 2014;5(1):2363-2373. doi:10.3762/bjnano.5.245

72. Dragicevic N, Maibach HI. Percutaneous Penetration Enhancers Chemical Methods in Penetration Enhancement: Nanocarriers. Percutaneous Penetration Enhanc Chem Methods Penetration Enhanc Nanocarriers. 2016:1-384.

73. Sreekumar S, Goycoolea FM, Moerschbacher BM, Rivera-Rodriguez GR. Parameters influencing the size of chitosan-TPP nano- and microparticles. Sci Rep. 2018;8(1):1-11. doi:10.1038/s41598-01823064-4

74. Cerqueira-Coutinho C, Santos-Oliveira R, Dos Santos E, Mansur CR. Development of a photoprotective and antioxidant nanoemulsion containing chitosan as an agent for improving skin retention. Eng Life Sci. 2015;15(6):593-604. doi:10.1002/elsc.201400154

75. Zhou M, Gan Y, Yang M, He C, Jia Y. Lipidomics analysis of facial skin surface lipids between forehead and cheek: association between lipidome, TEWL, and pH. J Cosmet Dermatol. 2020;(December 2019):1-7.

76. Sinico C, Manconi M, Peppi M, Lai F, Valenti D, Fadda AM. Liposomes as carriers for dermal delivery of tretinoin: in vitro evaluation of drug permeation and vesicle-skin interaction. $J$ Control Release. 2005;103(1):123-136. doi:10.1016/j.jconrel.2004.11.020

77. Gillet A, Compère P, Lecomte F, et al. Liposome surface charge influence on skin penetration behaviour. Int J Pharm. 2011;411(12):223-231. doi:10.1016/j.ijpharm.2011.03.049

78. Ogiso T, Yamaguchi T, Iwaki M, Tanino T, Miyake Y. Effect of positively and negatively charged liposomes on skin permeation of drugs. J Drug Target. 2001;9(1):49-59. doi:10.3109/ 10611860108995632

79. Khurana S, Jain NK, Bedi PMS. Nanoemulsion based gel for transdermal delivery of meloxicam: physico-chemical, mechanistic investigation. Life Sci. 2013;92(6-7):383-392. doi:10.1016/j. 1fs.2013.01.005 
80. Su R, Fan W, Yu Q, et al. Size-dependent penetration of nanoemulsions into epidermis and hair follicles: implications for transdermal delivery and immunization. Oncotarget. 2017;8(24):38214-38226. doi:10.18632/oncotarget. 17130

81. Elmataeeshy ME, Sokar MS, Bahey-El-Din M, Shaker DS. Enhanced transdermal permeability of terbinafine through novel nanoemulgel formulation; development, in vitro and in vivo characterization. Future J Pharm Sci. 2018;4(1):18-28. doi:10.1016/j.fjps.2017.07.003

82. Chantasart D, Kevin LS. Structure enhancement relationship of chemical penetration enhancers in drug transport across the stratum corneum. Pharmaceutics. 2012;4(1):71-92. doi:10.3390/ pharmaceutics4010071
83. Adib ZM, Ghanbarzadeh S, Kouhsoltani M, Khosroshahi AY, Hamishehkar H. The effect of particle size on the deposition of solid lipid nanoparticles in different skin layers: a histological study. Adv Pharm Bull. 2016;6(1):31-36. doi:10.15171/apb.2016.006

84. Ahmad S, Minhas MU, Ahmad M, Sohail M, Abdullah O, Badshah SF. Preparation and evaluation of skin wound healing chitosan-based hydrogel membranes. AAPS PharmSciTech. 2018;19(7):3199-3209. doi:10.1208/s12249-018-1131-z

\section{Publish your work in this journal}

The International Journal of Nanomedicine is an international, peerreviewed journal focusing on the application of nanotechnology in diagnostics, therapeutics, and drug delivery systems throughout the biomedical field. This journal is indexed on PubMed Central, MedLine, CAS, SciSearch ${ }^{\mathbb{R}}$, Current Contents ${ }^{\mathbb{R}} /$ Clinical Medicine, $^{-}$
Journal Citation Reports/Science Edition, EMBase, Scopus and the Elsevier Bibliographic databases. The manuscript management system is completely online and includes a very quick and fair peer-review system, which is all easy to use. Visit http://www.dovepress.com/ testimonials.php to read real quotes from published authors. 